
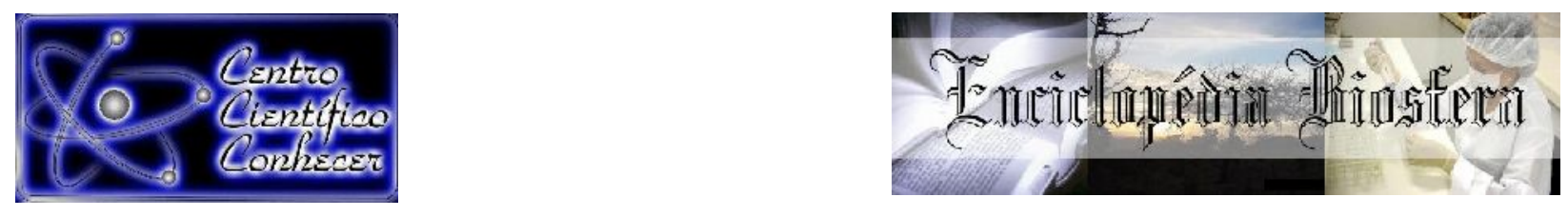

\title{
MACROFAUNA EDÁFICA INVERTEBRADA COMO BIOINDICADOR EM DOIS DIFERENTES FRAGMENTOS REMANESCENTES DE FLORESTA OMBRÓFILA MISTA NO PARANÁ
}

\author{
Everaldo dos Santos ${ }^{1}$, Vinícius Guimarães Toledo ${ }^{2}$, Heloísa Fernandes ${ }^{3}$, Leandro \\ Angelo Pereira ${ }^{4}$. \\ 1 Professor Doutor do Eixo Tecnológico de Meio Ambiente do Instituto Federal \\ do Paraná (everaldo.santos@ifpr.edu.br) Paraná-Brasil \\ 2 Discente de Tecnologia em Gestão Ambiental do Instituto Federal do Paraná, \\ Bolsista PIBIC. \\ 3 Professora Doutora do Eixo Tecnológico de Meio Ambiente do Instituto \\ Federal do Paraná \\ 4 Professor Doutor do Eixo Tecnológico de Meio Ambiente do Instituto Federal \\ do Paraná
}

Recebido em: 06/04/2019 - Aprovado em: 10/06/2019 - Publicado em: 30/06/2019

DOI: 10.18677/EnciBio_2019A82

\begin{abstract}
RESUMO
O trabalho teve como objetivo caracterizar a distribuição e funcionalidade da macrofauna edáfica invertebrada na interface solo-serapilheira em dois diferentes remanescentes de Floresta Ombrófila Mista. A área de estudo foi na cidade de Curitiba no primeiro Planalto do Paraná situada a uma altitude de 934,6 metros. Foram estudados dois fragmentos florestais nos diferentes Campi da Universidade Federal do Paraná (UFPR), o fragmento Trilha da Floresta (TF) no Campus Jardim Botânico, e o fragmento Bosque das Ciências Biológicas (BIO) no Campus Politécnico. A macrofauna edáfica foi coletada utilizando a metodologia Tropical Soil Biology and Fertility (TSBF). Foram coletados três monólitos de solo de $25 \times 25 \times 10 \mathrm{~cm}$ em um transecto de 50 metros. Coletou-se a serapilheira acumulada e os primeiros $10 \mathrm{~cm}$ de solo com gabarito. Os invertebrados da macrofauna edáfica foram triados e acondicionados para posterior identificação em nível de ordem taxonômica. Além disso, os exemplares foram classificados de acordo com a preferência trófica ou hábito alimentar. O litter coletado foi secado, triado em diferentes frações e pesado para posterior correlação com a fauna invertebrada. As análises estatísticas foram multivariadas utilizando o software PAST 2.0. A maior frequência relativa ocorreu no fragmento (BIO) tanto no solo como na serapilheira. E a ordem Oligochaeta foi maior no solo do fragmento (TF) seguido pela ordem Diplopoda. Na serapilheira o fragmento (BIO) apresentou maior frequência de Isopoda. A serapilheira apresentou maior diversidade de grupos pelo índice de Shannon $(H)$, equitabilidade de Simpson $(E)$ e riqueza $(S)$ quando
\end{abstract}


comparado com o solo nas duas áreas amostrais de fragmentos florestais. E a maior diversidade Shannon $(\mathrm{H})$ foi no fragmento $(\mathrm{BIO})$ devido a maior riqueza de grupos mostrando afinidade com o grupo trófico detritívoros-decompositores.

PALAVRAS-CHAVE: Bioindicadores, Biologia do solo. Invertebrados do solo.

\title{
EDAPHIC INVERTEBRATE MACROFAUNA AS A BIOINDICATOR IN TWO DIFFERENT FRAGMENTS OF OMBROPHILA FOREST REMANESCENT IN PARANÁ
}

\begin{abstract}
The objective of this work was to characterize the distribution and functionality of edaphic invertebrate macrofauna at the soil-litter interface in two different remnants of the Mixed Ombrophylous Forest. The study area was in the Curitiba in the first Plateau of Paraná located at an altitude of 934.6 meters. Two forest fragments were studied in the different Campuses of the Federal University of Paraná, the fragment Forest Path (TF) in the Botanical Garden Campus, and the Forest Biological Sciences (BIO) fragment in the Polytechnic Campus. The edaphic macrofauna was collected using Tropical Soil Biology and Fertility (TSBF) methodology. Three soil monoliths of $25 \times 25 \times 10 \mathrm{~cm}$ were collected on a 50 meter transect. The accumulated litter and the first $10 \mathrm{~cm}$ of soil with a template were collected. The invertebrates of the edaphic macrofauna were screened and conditioned for later identification at the taxonomic order level. In addition, the specimens were classified according to trophic preference or food habit. The collected litter was dried, screened in different fractions and weighed for posterior correlation with the invertebrate fauna. Statistical analyzes were multivariate using PAST 2.0 software. The highest relative frequency occurred in the fragment $(\mathrm{BIO})$ in both soil and litter. The order Oligochaeta was larger in the fragment soil (TF) followed by the Diplopoda order. In the litter in fragment (BIO) showed higher frequency of Isopoda. The litter presented a greater diversity of groups by the Shannon index $(H)$, Simpson equitability $(E)$ and richness $(S)$ when compared to the soil in the two sample areas of forest fragments. The greatest diversity Shannon $(\mathrm{H})$ was in the fragment $(\mathrm{BIO})$ due to the higher richness of groups showing affinity with the trophic group detritivores-decomposers.
\end{abstract}

KEYWORDS: Soil invertebrates, Bioindicators, Soil biology.

\section{INTRODUÇÃO}

A macrofauna invertebrada edáfica presente na interface solo-serapilheira do piso florestal da FOM, e outras formações florestais, desempenha um importante papel nos processos de funcionamento deste ecossistema pelo fato de ocuparem diferentes níveis tróficos, ou ainda, por estarem diretamente envolvidos nos processos de decomposição, ciclagem biogeoquímica, movimentação de material e transferência de nutrientes, interferindo nas características físicas e químicas do solo (MORSELLI, 2009; MEDEIROS et al., 2018).

Desta forma, o entendimento da diversidade e da dinâmica das populações que compõem esta fauna mostra-se como uma ferramenta útil na determinação da qualidade do ecossistema, pois é capaz de determinar impactos relevantes em ecossistemas submetidos a diferentes sistemas de cobertura vegetal, uso e 
degradação, tais como perda de fertilidade ou da capacidade produtiva do solo (BROWN et al., 2001; BARETTA et al., 2010; KLENK et al. 2014).

A fauna invertebrada do solo pode atuar como bioindicadora da qualidade ecossistêmica por meio de sua riqueza, diversidade, equitabilidade e funcionalidade (MELO et al., 2009; MARQUES et al., 2014; TRIANA et al., 2014; BIANCHI et al., 2017). A fragmentação dos habitats e a conversão de ecossistemas naturais em áreas urbanas ou de produção agrícola promovem a modificação da dinâmica de grupos que compõem a fauna invertebrada edáfica. GE. et al. (2014), em estudo com diferentes sistemas de cobertura na costa da China, mostraram que o uso do solo traz efeitos significativos na diversidade e composição da comunidade de macrofauna invertebrada. Ainda, Baretta et al. (2010) apresentaram possibilidades, em floresta com Araucária, do estabelecimento da correlação significativa entre macrofauna edáfica e variáveis ambientais de acordo com os atributos químicos e microbiológicos do solo.

Neste sentido, a cobertura vegetação pode ser diretamente relacionada com a qualidade edáfica, onde ecossistemas com maior complexidade de espécies vegetais como florestas nativas promovem melhores condições de umidade, matéria orgânica e $\mathrm{pH}$ do solo, oportunizando maior densidade e diversidade de grupos de invertebrados edáficos, os quais podem ser utilizados como bioindicadores, principalmente em áreas de recuperação e/ou regeneração ambiental (POMPEO, 2016; ARAÚJO et al. 2018; CORREIA et al., 2018).

No Paraná, a Floresta Ombrófila Mista (FOM) é uma unidade fitoecológica, componente do Bioma Mata Atlântica (IBGE, 2012) e Veloso et al. (1991), marcada pela presença de vegetação nativa do Primeiro Planalto do Paraná, tendo sido dominante na paisagem chegando a recobrir $37 \%$ da superfície do território paranaense nos períodos antecedentes aos processos de ocupação e urbanização, restando atualmente apenas $0,8 \%$ de seus remanescentes naturais (FUPEF, 2003). A FOM é caracterizada pela coexistência de representantes de floresta tropical e temperada, com predominância da Araucaria angustifolia (Bertol.) Kuntze, popularmente denominado Pinheiro-do-Paraná. Esta espécie típica é exclusiva dos planaltos da região Sul do Brasil, e apresenta particularidades como ocorrência entre 800 e 1200 m, podendo ainda ocorrer em regiões de maior altitude (RODERJAN et al., 2002; MARTINS et al. 2017).

Partindo-se do pressuposto que a FOM apresentam diferentes níveis de diversidade de espécies vegetais e condições particulares de composição da macrofauna edáfica, as quais atribui-se papel chave na regeneração natural deste ecossistema, e ainda, pelo fato dos diferentes fragmentos florestais poderem apresentar diferentes níveis de diversidade, riqueza e densidade de grupos da fauna edáfica, o presente trabalho teve como objetivo caracterizar a distribuição e funcionalidade da macrofauna edáfica invertebrada na interface solo-serapilheira em dois diferentes remanescentes de Floresta Ombrófila Mista localizados no município de Curitiba, PR. 


\section{Área de Estudo}

\section{MATERIAL E MÉTODOS}

A área de estudo está localizada na cidade de Curitiba, no primeiro Planalto do Paraná, situada a uma altitude de 934,6 metros com coordenadas centrais, latitude $25^{\circ} 25^{\prime} 40^{\prime \prime} \mathrm{S}$ e longitude $49^{\circ} 16^{\prime} 23^{\prime \prime} \mathrm{W}$. O clima, de acordo com a classificação de Köppen, é caracterizada como Cfb, subtropical úmido, mesotérmico, sem estação seca, com verões frescos e invernos com geadas frequentes (IPPUC, 2019).

A localização dos fragmentos florestais onde foram feitas as amostragens localizam-se nos diferentes Campi da Universidade Federal do Paraná (UFPR), o Campus Centro Politécnico, com o fragmento florestal Trilha da Floresta (TF) e Campus Jardim Botânico, com o fragmento Bosque das Ciências Biológicas (BIO). Os dois fragmentos ou áreas são separadas pelo trecho urbano do antigo traçado da rodovia BR 116 hoje chamada de BR 476 ou também conhecida como Linha Verde (Figura 1).

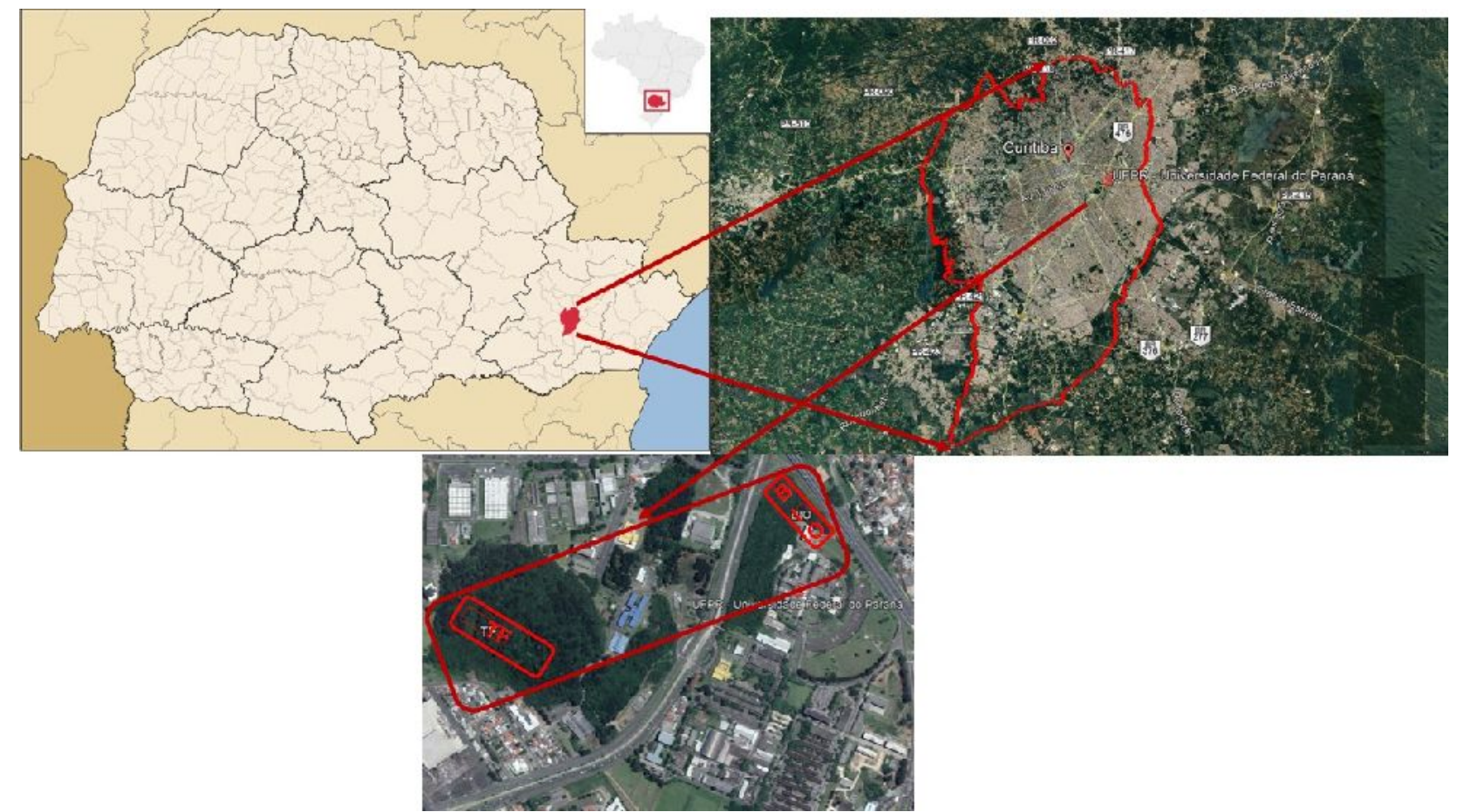

FIGURA 1. Localização da área de estudo. Destaque para os dois fragmentos florestais ou unidades amostrais denominadas Trilha da Floresta (TF) e Bosque das Ciências biológicas (BIO), nos diferentes campi da Universidade Federal do Paraná (UFPR).

\section{Coleta, amostragem, identificação e análise}

A macrofauna edáfica foi coletada utilizando a metodologia proposta pelo Tropical Soil Biology and Fertility TSBF (ANDERSON; INGRAM, 1993) adaptado. Três monólitos de solo de $25 \times 25 \times 10 \mathrm{~cm}$ foram coletados em um transecto de 50 metros com auxílio de um gabarito, em cada fragmento florestal, denominado Trilha da Floresta (TF) e Bosque das Ciências Biológicas (BIO). O solo foi coletado nos primeiros 10 centímetros $(0-10 \mathrm{~cm})$ da superfície devido à alta compactação abaixo 
desta profundidade. Cada monólito coletado foi fracionado manualmente em serapilheira e solo.

Os monólitos coletados foram embalados em sacos plásticos e transportados para laboratório e triados, posteriormente e imediatamente após a coleta. Em laboratório, com o auxílio de pinças e lupas de mão, foi realizada a extração e triagem dos exemplares de invertebrados da macrofauna edáfica, nos monólitos provenientes das amostras de solo e serapilheira, com esforço amostral de aproximadamente 30 minutos por amostra. Os invertebrados extraídos foram acondicionados em frascos contendo álcool $70 \%$, para conservação e posterior identificação, que se deu em grandes táxons ao nível de ordem taxonômica (Dos SANTOS et al., 2015).

Após o levantamento de indivíduos por grupo foi avaliada a funcionalidade ecológica da macrofauna invertebrada determinando-se o número de indivíduos por grupo (táxon) em cada $\mathrm{m}^{2}$ e a frequência relativa (\%) em cada sistema. Foram determinados ainda os índices de diversidade de Shannon $(H)$, equitabilidade de Simpson (E), dominância de Berger-Parker (D) e riqueza (S). A categorização dos invertebrados foi realizada conforme proposto por Merlim, (2005) e Moço et al., (2005), adaptado. Assim, os grupos funcionais, de acordo com os hábitos alimentares ou função trófica foram: Hymenoptera sociais, Isoptera sociais, decompositores-detritívoros, predadores e geófagos.

Quanto à serapilheira ou litter, após coletadas e triadas, estas foram submetidas à secagem em estufa de circulação forçada, a temperatura de $65^{\circ} \mathrm{C}$. Depois da secagem, o litter acumulado foi separado nas frações: Folhas Recém depositadas (FR), Folhas em Decomposição (FD), Ramos Finos (RF) (<2cm de diâmetro), órgãos reprodutivos (Flores, Frutos e Sementes) (FFS) e a fração Peneira $(P)$, também chamada de miscelânea, composta de fragmentos finos que foram tamisados em peneira com malha de $4 \mathrm{~mm}$. Posteriormente, o material foi pesado em balança analítica. As folhas foram ainda separadas por morfotipos, afim de distinguir as diferentes espécies de vegetais componentes da serapilheira.

\section{Análises estatísticas}

Os dados coletados foram ordenados e processados com o uso de software específico. Para os índices de diversidade, equitabilidade, dominância e riqueza foi utilizado o software Dives v2.0 (RODRIGUES, 2007). Os dados de grupos taxonômicos de macrofauna invertebrada, hábito alimentar, biomassa e frações da serapilheira e índices populacionais foram analisados de forma não parametrizada pela Análise de Componentes Principais (ACP) utilizando o software PAST 2.0 (HAMMER ,2001).

\section{RESULTADOS E DISCUSSÃO}

Os dados encontrados apontam alguns resultados relacionados a frequência relativa e distribuição de indivíduos por $\mathrm{m}^{2}$ nas diferentes ordens nos deferentes compartimentos solo e serapilheira nos fragmentos Trilha da Floresta (TF) e Bosque das Ciências Biológicas (BIO). De forma geral a maior frequência foi no fragmento (BIO) tanto no solo como na serapilheira. Observou-se que no solo, o fragmento (TF) teve maior frequência relativa de Oligochaetas $(43,7 \%)$ seguido da ordem 
Diplopoda (18,7\%).

O fragmento florestal (BIO) apresentou maior frequência de Isopoda $(46,2 \%)$, seguido de Formicidae (18,5\%). Dos Santos et al. (2015) também encontraram maiores frequências relativas de Oligochaetas em solo de agrofloresta e floresta nativa em Floresta Ombrófila Densa. Marques et al. (2014) também encontraram maiores frequências em solo em diferentes coberturas vegetais com predominância na mata nativa. Em (TF) as Oligochaetas apresentaram maior frequência em relação a $(\mathrm{BIO})$ o que pode indicar maior afinidade das espécies desta ordem com as características deste solo, que pode ser diferente do solo do fragmento (BIO).

Rosa et al. (2015) encontraram elevada correlação com grupos da macrofauna e os teores de $\mathrm{MO}, \mathrm{Ca}, \mathrm{Mg}, \mathrm{P}, \mathrm{Al}, \mathrm{K}$ e bioporos, ou, estabelecimento da macrofauna edáfica nos diferentes sistemas de uso e manejo. Além disso, Triana et al. (2014) encontraram correlações positivas com Oligochaetas e florestas mais maduras com melhores fluxos de nutrientes. Quanto as altas frequências das ordens Isopodas e Diplopodas, mostram a presença de detritívoros e decompositores no solo conforme Triana et al. (2014).

Quanto à frequência relativa no compartimento serapilheira, o fragmento (TF) apresentou $20 \%$ de indivíduos das Coleoptera e Oligochaeta e 13,3\% de Isoptera e Diplopoda, enquanto BIO apresentou maior frequência de Isopoda com 46,2\% seguido de Formicidae com 18,5\%. Da mesma forma que no solo a serapilheira ou litter, apresentou altas frequências das ordens Isopoda e Diplopoda mostrando que os detritívoros e decompositores tem preferência pela serapilheira, estas informações corroboram com Baretta et al. (2010) e Triana et al. (2014).

Além disso, Scoriza e Correia (2016) e Pinheiro et al. (2014) ao avaliarem a eficiência da fauna edáfica como indicadora de conservação de fragmentos de Floresta Ombrófila Densa, constataram que a serapilheira estocada no solo está relacionada com a riqueza média e a atividade da fauna do solo, e a relação positiva da ordem Coleóptera com a serapilheira depositada no solo (Tabela 1).

TABELA 1- Distribuição de grupos em ordem taxonômicas com valores de frequência relativa (\%) e indivíduos por $\mathrm{m}^{2}$ no solo e serapilheira nas unidades amostrais Trilha da Floresta (TF) e Bosque das Ciências Biológicas (BIO).

\begin{tabular}{|c|c|c|c|c|c|c|c|c|}
\hline \multirow[b]{3}{*}{$\begin{array}{l}\text { Grupo } \\
\text { Ordem }\end{array}$} & \multicolumn{4}{|c|}{ SOLO } & \multicolumn{4}{|c|}{ SERAPILHEIRA } \\
\hline & \multicolumn{2}{|c|}{ TF } & \multicolumn{2}{|c|}{ BIO } & \multicolumn{2}{|c|}{ TF } & \multicolumn{2}{|c|}{ BIO } \\
\hline & $\begin{array}{l}\text { Ind. } \\
\mathrm{m}^{2}\end{array}$ & $\begin{array}{c}\text { F. R } \\
\%\end{array}$ & $\begin{array}{l}\text { Ind. } \\
\mathrm{m}^{2}\end{array}$ & $\begin{array}{c}\text { F. R } \\
\%\end{array}$ & $\begin{array}{l}\text { Ind. } \\
\mathrm{m}^{2}\end{array}$ & $\begin{array}{c}\text { F. R } \\
\%\end{array}$ & $\begin{array}{l}\text { Ind. } \\
\text { m² }^{2}\end{array}$ & $\begin{array}{c}\text { F. R } \\
\%\end{array}$ \\
\hline Formicidae & 0 & 0 & 74,6 & 18,5 & 5,33 & 6,66 & 53,3 & $(18,5$ \\
\hline Isoptera & 0 & 0 & 0 & 0 & 10,6 & 13,3 & 0 & 0 \\
\hline Diplopoda & 0 & 0 & 0 & 0 & 10,6 & 13,3 & 0 & 0 \\
\hline Coleoptera & 5,33 & 6,25 & 48 & 5,55 & 16 & 20 & 16 & 5,55 \\
\hline Oligochaeta & 37,3 & 43,7 & 5,33 & 1,85 & 16 & 20 & 5,33 & 1,85 \\
\hline Blattodea & 0 & 0 & 0 & 3,7 & 0 & 0 & 10,6 & 3,7 \\
\hline Decapoda & 0 & 0 & 5,33 & 1,85 & 0 & 0 & 5,33 & 1,85 \\
\hline Isopoda & 0 & 0 & 5,33 & 46,2 & 0 & 0 & 133,3 & 46,2 \\
\hline
\end{tabular}




\begin{tabular}{|c|c|c|c|c|c|c|c|c|}
\hline Homoptera & 0 & 0 & 0 & 3,7 & 5,33 & 6,66 & 10,6 & 3,7 \\
\hline Chilopoda & 0 & 0 & 5,33 & 0 & 0 & 0 & 0 & 0 \\
\hline Aranea & 0 & 0 & 0 & 5,55 & 0 & 0 & 16 & 5,55 \\
\hline Mollusca & 0 & 0 & 0 & 3,7 & 0 & 0 & 10,6 & 3,7 \\
\hline Thysanura & 0 & 0 & 10,6 & 0 & 0 & 0 & 0 & 0 \\
\hline Orthoptera & 0 & 0 & 0 & 1,85 & 0 & 0 & 5,33 & 1,85 \\
\hline Diplopoda & 16 & $(18,7)$ & 0 & 1,85 & 5,33 & 6,66 & 5,33 & 1,85 \\
\hline Dermaptera & 0 & 0 & 0 & 3,7 & 0 & 0 & 10,6 & 3,7 \\
\hline $\begin{array}{l}\text { Juvenil não } \\
\text { identificado }\end{array}$ & 26,6 & 31,2 & 37,3 & 1,85 & 10,6 & 13,3 & 5,33 & 1,85 \\
\hline Total & 85,3 & 100 & 192 & 100 & 80 & 100 & 288 & 100 \\
\hline
\end{tabular}

A tabela 2 apresenta os resultados obtidos para os diferentes índices ecológicos avaliados. Percebe-se que a serapilheira apresentou maior diversidade de grupos pelo índice de Shannon $(H)$, equitabilidade de Simpson (E) e riqueza (S) quando comparado com o solo nas duas áreas amostrais de fragmentos florestais. No solo destaca-se a maior equitabilidade de grupos e menor dominância em TF. Esperava-se que a área TF tivesse apresentado maior índice de diversidade de Shannon $(\mathrm{H})$ por causa da vegetação com maior número de araucárias, embora os índices de equitabilidade de Simpson (E) tenha sido maior em TF e o índice de Berger-Parker (D) foi menor, mostrando maior equitabilidade e menor dominância. A diversidade Shannon $(\mathrm{H})$ foi maior na área $\mathrm{BIO}$ devido a maior riqueza de grupos.

Quanto a unidade amostral ou fragmento florestal, observou-se que a diversidade de Shannon $(\mathrm{H})$ foi maior no Bosque das Ciências Biológicas (BIO) quando comparado com a Trilha da Floresta (TF). Moço et al. (2005) e Armendano et al. (2018) observaram que não há diferença na densidade e riqueza da macrofauna edáfica em florestas com diferentes níveis de conservação, mas mostrou diferença com pastagem e monoculturas como Eucaliptus sp. ou tomates cultivados de forma tradicional. Por outro lado, Copatti e Daudt (2009) evidenciaram a maior abundância em mata nativa quando comparado com monocultura de Pinus elliottii, da mesma forma, diversidade e riqueza foram superiores para este ambiente também, mostrando que a mata nativa possui fatores favoráveis aos organismos, como maior disponibilidade de recursos, homeostase do meio e maior diversidade de espécies vegetais.

TABELA 2 - Valores dos Índices de Diversidade de Shannon (H), Dominância de BergerParker (D), Equitabilidade de Simpson (E) e Riqueza de grupos (S) no solo e litter nos fragmentos florestais Trilha da Floresta (TF) e Bosque das Ciências Biológicas (BIO).

\begin{tabular}{c|c|c|c|c}
\hline \multirow{2}{*}{ Índice } & \multicolumn{2}{|c|}{ SOLO } & \multicolumn{2}{c}{ SERAPILHEIRA } \\
\cline { 2 - 5 } & TF & BIO & TF & BIO \\
\hline Shannon (H) & 0,71 & 0,83 & 1,73 & 2,17 \\
\hline
\end{tabular}




\begin{tabular}{c|c|c|c|c}
\hline $\begin{array}{c}\text { Berger-Parker } \\
\text { (D) }\end{array}$ & 0,36 & 0,43 & 0,36 & 0,51 \\
\hline Simpson (E) & 0,56 & 0,51 & 0,98 & 0,80 \\
\hline Riqueza (S) & 3 & 7 & 7 & 13
\end{tabular}

Considerando os hábitos alimentares ou função trófica observou-se que na serapilheira o grupo decompositores-detritívoros teve a maior frequência relativa, seguido de Hymenoptera sociais nos dois fragmentos florestais BIO e TF. No solo a maior frequência foi de Hymenoptera sociais, seguido de decompositoresdetritívoros. Triana et al. (2014) e Pinheiro et al. (2014) também encontraram uma maior frequência de decompositores e detritívoros em serapilheira quando comparado com o solo. Menezes et al. (2009) Encontraram relação entre decompositores e detritívoros em diferentes estágios sucessionais.

Destaca-se que o solo teve maior frequência de geófagos, principalmente em TF. Este fato se deve a alta frequência de Oligochaetas no solo do fragmento TF. Kalu et al. (2015) encontraram maior densidade populacional de minhocas em ambientes menos perturbados com maior umidade e matéria orgânica. Este fato pode explicar maior frequência em TF em relação ao fragmento $\mathrm{BIO}$, pois o solo neste fragmento aparentemente tinha maior umidade (Figura 1). Além disso, Jouquet et al. (2006) destacam que o grupo Oligochaeta são engenheiros de ecossistemas e desempenham papel importante na estruturação física do solo estando diretamente associado a vegetação.

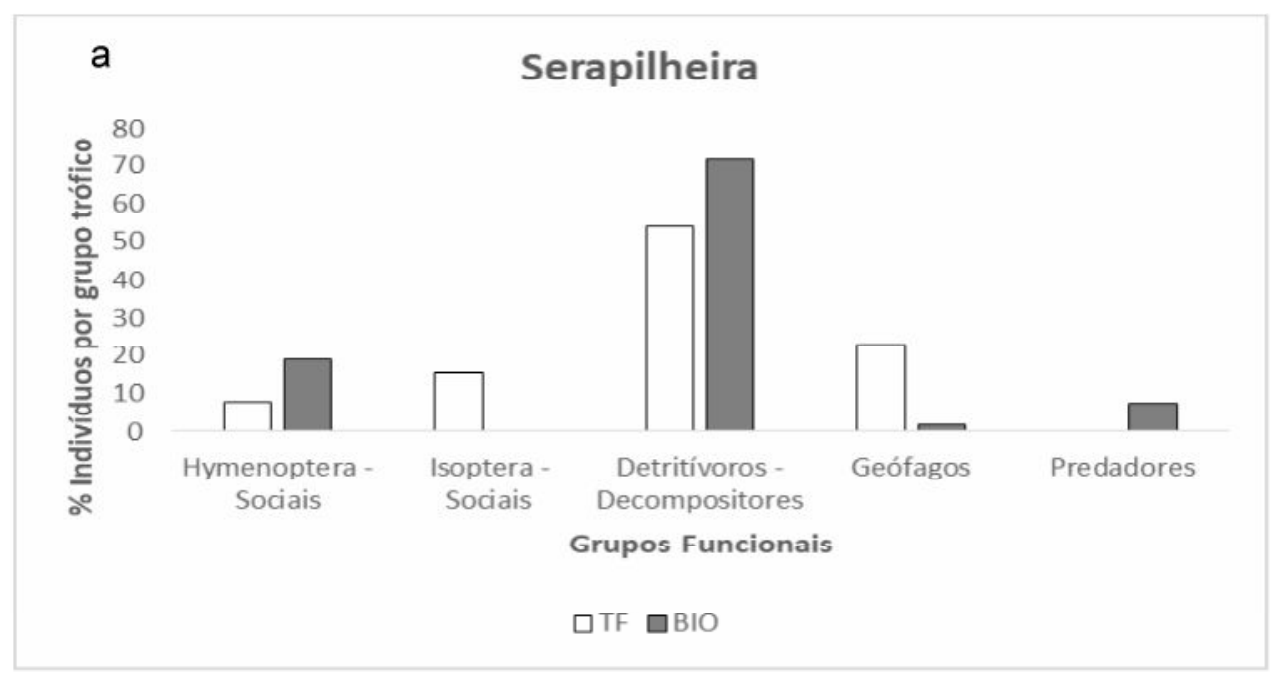




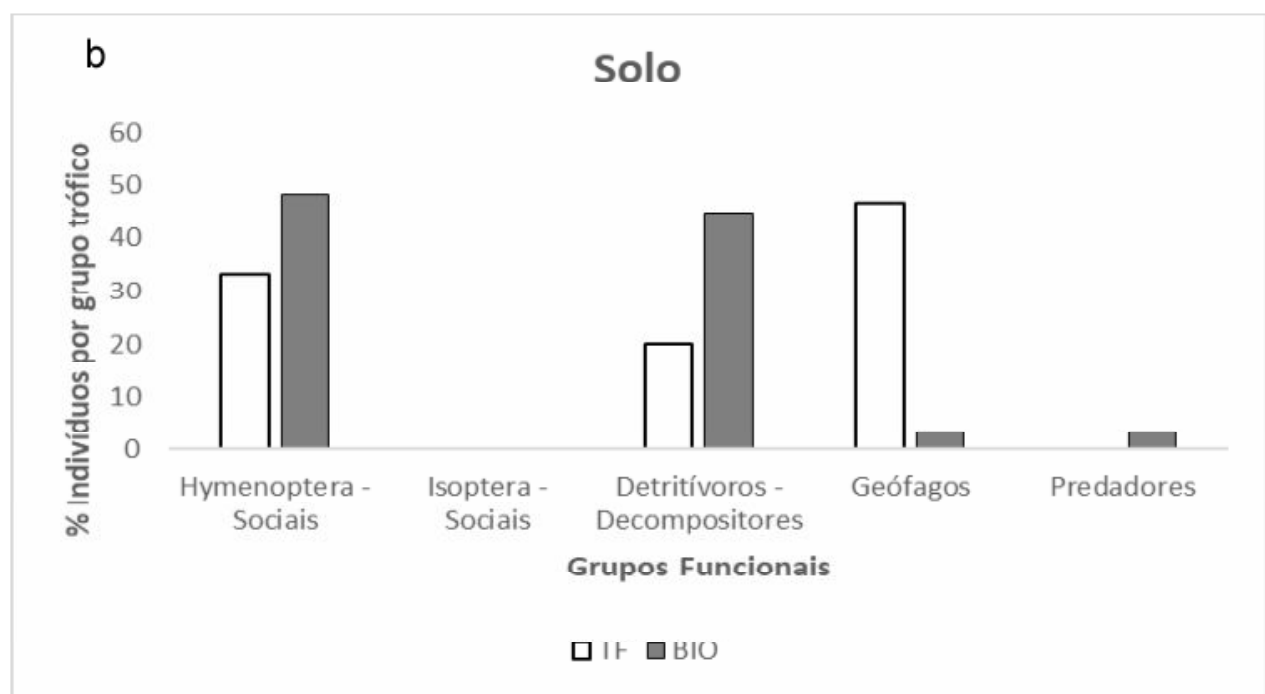

FIGURA 1 a) Grupos de macrofauna invertebrada de acordo com o hábito alimentar ou nível trófico na serapilheira ou litter b) Grupos de macrofauna invertebrada de acordo com o hábito alimentar ou nível trófico no solo.

A análise de componentes principais (ACP) foi realizada utilizando-se os resultados obtidos dos grupos funcionais de acordo com hábito alimentar, correlacionando-os com riqueza, diversidade, equitabilidade e dominância nos diferentes compartimentos (Solo - Soil e Serapilheira - Litter). Os resultados apresentaram $81,1 \%$ de correlação com a ACP1 que mostrou 57,8\% de correlação e a ACP2 com 33,3\%.

A componente principal 1 mostrou maior diversidade de Shannon e riqueza na serapilheira (Litter) da unidade amostral Bosque das Ciências Biológicas (BIO) correlacionando-se com o grupo funcional decompositores-detritívoros e predadores. Mostrou também maior dominância de grupos indicado pelo índice de Berger-Parker (D) (Figura 2). A ACP2 mostrou correlação entre Geófagos e Hymenoptera Sociais no compartimento solo das diferentes unidades amostrais. Houve maior afinidade da macrofauna invertebrada pela serapilheira. A macrofauna edáfica é influenciada pelos atributos químicos e físicos do solo, onde a dispersão e estabelecimento desses organismos pode explicar as variações de grupos de organismos de acordo com sistema de cobertura e uso do solo Rosa et al. (2015). Além de características físicas e químicas do solo e da serapilheira que podem determinar maior afinidade dos grupos com os diferentes compartimentos conforme afirmam Marques et al. (2014) e Ge et al. (2014) e Kalu et al., (2015). 


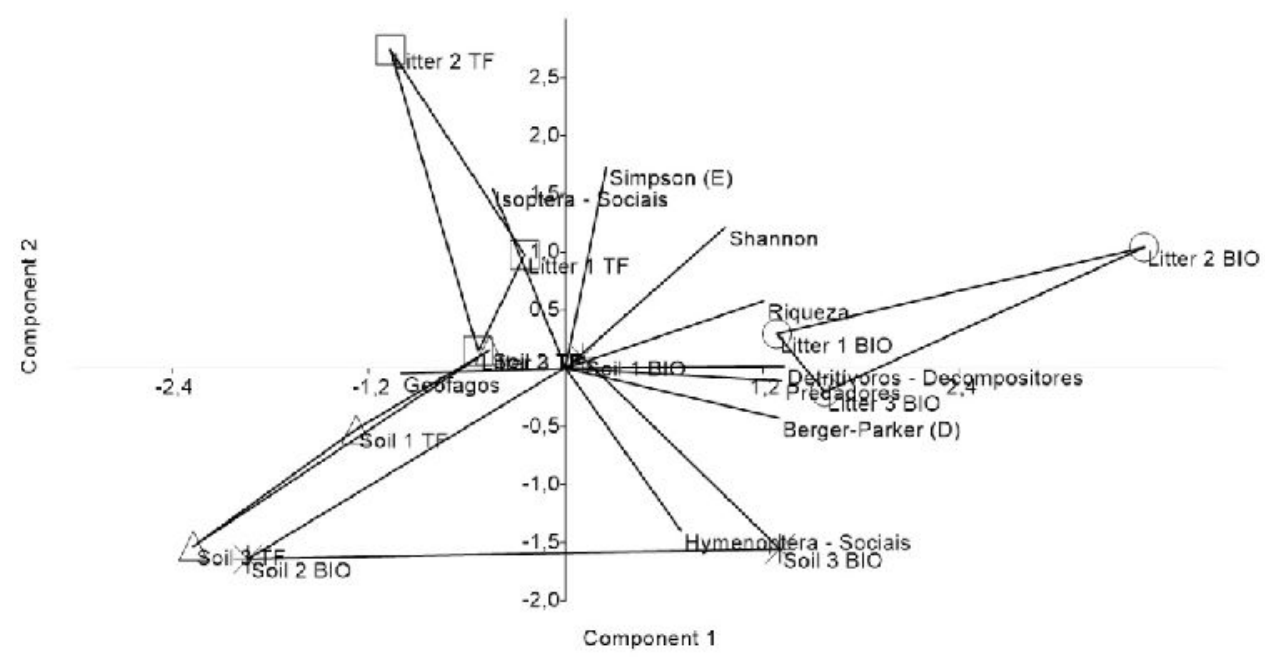

FIGURA 2 Gráfico de Análise de Componentes Principais (ACP), mostrando correlação entre solo e litter nos dois fragmentos florestais Bosque das Ciências Biológicas (BIO) e Trilha da Floresta (TF) com os índices de diversidade de Shannon, equitabilidade de Simpson (E), dominância de Berger-Parker (D), riqueza e grupos de acordo com a função trófica Isoptera sociais, Hymenoptera sociais, Detritívoros-Decompositores, Predadores e Geófagos.

A análise de componentes principais (ACP), apresentada na Figura 4, mostra a correlação entre o grupo trófico detritívoros-decompositores e o índice de diversidade de Shannon $(\mathrm{H})$ da macrofauna edáfica com atributos da serapilheira acumulada como a biomassa total e biomassa das frações folhas em decomposição (FD) e peneira (P) além da riqueza das espécies vegetais que compõem o litter ou serapilheira. Os resultados mostraram correlação de 53,9\% da ACP 1 e 22,1 da ACP 2 mostrando um total de 76\% de correlação. Onde a ACP 1 mostrou correlação entre a diversidade de Shannon $(\mathrm{H})$ com o grupo detritívoros-decompositores na fração folhas em decomposição (FD) no litter do fragmento BIO.

A ACP 2 mostrou correlação entre a biomassa total do litter com a riqueza de espécies que compõem este material nas frações peneira $(P)$, que é o material mais fragmentado, e fração ramos finos (RF) no fragmento TF. Dessa forma, pode-se perceber que os detritívoros decompositores podem promovem maiores taxas de decomposição e que a fração do litter que mostra maior afinidade com este grupo são as folhas em decomposição (FD). E no fragmento TF deve ter maior acúmulo de litter devido a maior biomassa total e das frações P e RF. Frasson et al. (2016) encontrou relação significativa entre a riqueza de espécies da macro e meso fauna invertebrada em áreas mais sombreadas e com maior área de vegetação com aumento da taxa de decomposição da serapilheira. De acordo com Pinheiro et al. (2014) e Mueller et al. (2015) a decomposição do litter pode ter relação direta com as espécies que compõem o material e podem direcionar a ação dos detritívoros e decompositores. 


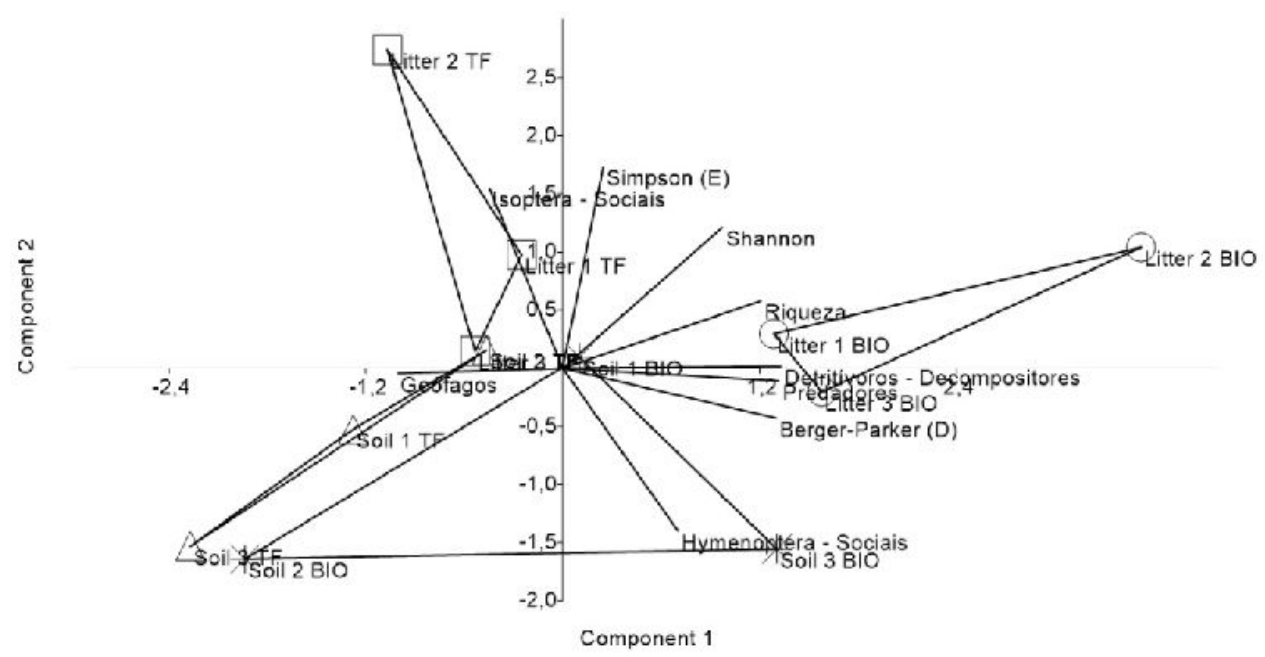

FIGURA 3 Gráfico de Análise de Componentes Principais (ACP), mostrando correlação entre litter nos dois fragmentos florestais Bosque das Ciências Biológicas (BIO) e Trilha da Floresta (TF) com a Biomassa total do litter e o índice de diversidade de Shannon $(\mathrm{H})$, grupo funcional de acordo com a função trófica Detritívoros-Decompositores, frações da serapilheira ou litter Peneira (P), Ramos Finos (RF), Folhas em Decomposição (FD) e Riqueza de spp vegetais que compõem o litter (Riqueza Litter).

A biomassa total de serapilheira foi maior no fragmento florestal ou unidade amostral TF com 22484,8 Kg.h-1 quando comparado com o fragmento BIO. Comparando as diferentes frações da serapilheira, observou-se que a maior produção de biomassa foi na fração Peneira $(P)$, seguido da fração ramos finos RF tanto em TF como no fragmento BIO (Tabela 3). Destaca-se a maior produção de material na fração Peneira $(P)$ da serapilheira do fragmento florestal TF.

A maior biomassa total e maior biomassa da fração $P$ provavelmente se deve a composição florística do fragmento que pode influenciar no material a ser estocado na serapilheira devido as espécies que compõem o litter acumulado no piso dos fragmentos florestais (INKOTTE et al., 2017). De acordo com Araújo et al. (2018) maior diversidade de espécies vegetais e consequentemente maior produção de serapilheira, promove um ambiente favorável para o desenvolvimento e manutenção do ecossistema, aumentando o índice de decompositores, promovendo inter-relações de antibiose e simbiose, tornando o ecossistema mais rico.

TABELA 3 - Biomassa da serapilheira (Kg.h-1) acumulada presente nas frações coletadas das diferentes áreas amostrais (TF e BIO): Folhas Recém depositadas (FR), Folhas em Decomposição (FD), Ramos Finos (RF), fração Peneira (P) e Flores, Frutos e Sementes (FFS) e Biomassa total.

\begin{tabular}{lllllll}
\hline $\begin{array}{l}\text { Unidade } \\
\text { amostral/Biomassa } \\
\text { em Kg. }{ }^{-1}\end{array}$ & FR & FD & RF & P & FFS & $\begin{array}{c}\text { Biomassa } \\
\text { Total }\end{array}$ \\
\hline Serapilheira TF & 604,8 & 289,0 & $5.100,2$ & $16.233,6$ & 257,3 & $22.484,8$ \\
\hline Serapilheira BIO & 257,0 & 544,0 & $2.932,8$ & $10.383,4$ & 322,1 & $14.439,4$ \\
\hline
\end{tabular}




\section{CONCLUSÕES}

Os resultados encontrados no presente trabalho apontam uma maior frequência relativa ocorreu no fragmento $(\mathrm{BIO})$ tanto no solo como na serapilheira. $\mathrm{E}$ a ordem Oligochaeta foi maior no solo do fragmento (TF) seguido pela ordem Diplopoda. O fragmento florestal (BIO) apresentou maior frequência de Isopoda. $\mathrm{Na}$ serapilheira o fragmento $(\mathrm{BIO})$ apresentou maior frequência de Isopoda.

Além disso, seria possível dizer que a serapilheira apresentou maior diversidade de grupos pelo índice de Shannon $(H)$, equitabilidade de Simpson $(E)$ e riqueza (S) quando comparado com o solo nas duas áreas amostrais de fragmentos florestais. E a maior diversidade Shannon $(\mathrm{H})$ foi no fragmento $(\mathrm{BIO})$ devido a maior riqueza de grupos mostrando afinidade com o grupo trófico detritívorosdecompositores.

De maneira geral a macrofauna invertebrada do solo mostrou diferente funcionalidade nos dois fragmentos florestais devido as características edáficas em cada fragmento, onde a serapilheira acumulada provavelmente direcionou as diferentes condições físicas, químicas e biológicas dos ecossistemas.

\section{AGRADECIMENTOS}

Agradecemos ao Conselho Nacional de Desenvolvimento Científico e Tecnológico (CNPq) pela concessão da Bolsa de Pesquisa e pelo apoio financeiro que muito tem nos auxiliado na realização das nossas pesquisas.

\section{REFERÊNCIAS}

AARMENDANO, A., ROUAUX, J., SALAZAR MARTÍNEZ, A. Fauna edáfica asociada a cultivos hortícolas convencionales de tomate en La Plata (Buenos Aires, Argentina). Acta Zoológica Mexicana (nueva serie), 34, 1-12. 2018 DOI: https://doi.org/10.21829/azm.2018.3412116

ANDERSON, J. M.; INGRAM, J. S. I. Tropical soil biology and fertility: a handbook of methods. Wallingford: CAB International, ed. 2, 1993.

ARAUJO, E. C. G.; SILVA, T. C.; LIMA, T. V.; SANTOS, N. A. T.; BORGES, C. H. A. Macrofauna como bioindicadora de qualidade do solo para agricultura convencional e agroflorestal. Agropecuária Científica no Semiárido. V. 14, n.2. 108-116. 2018. Disponível em: http://revistas.ufcg.edu.br/acsa/index.php/ACSA/article/view/975

BARETTA, D.; BROWN, G. G.; CARDOSO, E. J. B. N. Potencial da macrofauna e variáveis edáficas como indicadores da qualidade do solo em áreas com Araucaria. Acta Zoológica Mexicana. n. especial 2. 35-50 p. 2010. Disponível em: www.scielo.org.mx/pdf/azm/v26nspe2/v26nspe2a10.pdf

BIANCHI, M. O.; SCORIZA, R. N.; NOGUEIRA, R.; RESENDE, A. S.; CAMPELLO, E. F. C.; CORREIA, M. E. F.; SILVA, E. M. R. Soil Macrofauna as Indicators in Tree Legume Revegetation. FLORAM. v. 24. 2017. DOI:

http://dx.doi.org/10.1590/2179-8087.085714

BROWN, G.G.; FRAGOSO, C.; BAROIS, I.; ROJAS, P.; PATRÓN, 
J. C.; BUENO, J.; MORENO, A.G.; LAVELLE, P.; ORDAZ, V.; RODRíGUEZ, C. Diversidad y rol funcional de la macrofauna edáfica em los ecosistemas tropicales mexicanos. Acta Zoológica Mexicana. 1: 79-110. 2001. Disponível em: https://www.redalyc.org/articulo.oa?id=57500006

COPATTI, C. E.; DAUDT, C. R. Diversidade de artrópodes na serapilheira em fragmentos de mata nativa e Pinus elliottii (Engelm. Var elliottii). Ciência e Natura. v.31, n.1. 95 - 113 p. 2009. Disponível em:

https://periodicos.ufsm.br/cienciaenatura/article/viewFile/9913/5952

CORREIA, D. S., PASSOS, S. R., PROENÇA, D. N., MORAIS, P. V., XAVIER, G. R., CORREIA, M. E. F. Microbial diversity associated to the intestinal tractof soil in vertebrates. Applied Soil Ecology. n. 131 p. 38-46. 2018.

DOI: https://doi.org/10.1016/j.apsoil.2018.07.009

DOS SANTOS, E. SANTOS, R. C.; MARQUES, R. Macrofauna edáfica na interface solo-serrapilheira e a relação com atributos químicos de um Espodossolo sob dois diferentes sistemas de conservação e uso do solo no município de Paranaguá-PR. Enciclopédia Biosfera. v.11 n.21. 2015. Disponível em: http://www.conhecer.org.br/enciclop/2015b/biologicas/macrofauna\%20edafica.pdf

FRASSON, J.M. F.; ROSADO, J. L. O.; ELIAS, S. G.; HARTER-MARQUES, B.Litter decomposition of two pioneer tree species and associated soil fauna in areas reclaimed after surface coal mining in Southern Brazil. Revista Brasileira de Ciência do Solo. v. 40. 2016. http://dx.doi.org/10.1590/18069657rbcs20150444

FUPEF. Fundação de Pesquisas Florestais do Paraná. Diagnóstico da Cobertura Vegetal da Área Proposta para a Construção da PCH São Jerônimo e do Contexto Vegetacional do Seu Entorno. Curitiba: BRASCAN/FUPEF, 2003.

GE, B.; ZHANG, D.; TANG, B.; ZHOU, C. Effect of land cover on biodiversity and composition of a soil macrofauna community in a reclaimed coastal area at Yancheng, China. Turkish Journal of Zoology. 38: 229-233, 2014.

DOI:10.3906/zoo-1302-37

HAMMER, O.; HARPER, D.A.T.; RYAN, P. D. Paleontological statistics software package for education and data analysis. Paleontological Data Analysis. Blackwell. 2001. PAST.

IBGE. Instituto Brasileiro de Geografia e Estatística. Manual técnico da vegetação brasileira. (Série manuais técnicos em geociências, n. 1). $2^{\underline{a}}$ ed. Rio de Janeiro, 271p.2012.

INKOTTE, J.; MAFRA, A. L.; RIOS, P. D.; BARETTA, D.; VIEIRA, H. C. Litter fall deposition in eucalyptus plantations and native forests in the highland and west regions of Santa Catarina, Southern Brazil. Scientia Forestalis. v. 43, n. 106, p. 261-270, jun. 2015. Disponível em: 
https://www.ipef.br/publicacoes/scientia/nr106/cap02.pdf

IPPUC - Instituto de Pesquisa e Planejamento Urbano de Curitiba. Curitiba em Dados. [cited 2019 abr. Availablefrom:http://www.ippuc.org.br/Bancodedados/Curitibaemdados/Curitiba_em_ dados_Pesquisa.htm

JOUQUET, P.; DAUBER, J.; LAGERLÖF, J.; LAVELLE, P.; LEPAGE, M. Soil invertebrates as ecosystem engineers: intended and accidental effects on soil and feedback loops. Applied Soil Ecology. 32: 153-164. 2006.

DOI: 10.1016/j.apsoil.2005.07.004

KALU, S.; KOIRALA, M.; KHADAKA, U. R. Earthworm population in relation to different land use and soil characteristics. Journal of Ecology and the Natural Environment. v. 7, n. 5. 124-131p. 2015. DOI: 10.5897/JENE2015.0511

KLENK, L. A.; ZAEDANEACK, M.A.C.; MOTTA, A.C.V.; CONSALTER, R.; RICHETER, A.S.; BORGES, E. Macrofauna invertebrada edáfica em pastagem sul brasileira sob diferentes preparos orgânicos. Comunicata Scientiae. v.5, n.3, p.339-348, 2014.

MARQUES, D. M.; SILVA, A. B.; SILVA, L. M.; MOREIRA, E. A.; PINTO, G. S. Edaphic macrofauna in different plant covers. Bioscience. Journal. Uberlandia, v. 30, n. 5, p. 1588-1597, Sept./Oct. 2014. Disponível em:

https://www.researchgate.net/publication/289853483_Edaphic_macrofauna_in_differ ent_plant_covers

MARTINS, P. J.; MAZON, J. A.; MARTINKOSKI, L.; BENIN, C. C.; WATZLAWICK, L. F. Dinâmica da Vegetação Arbórea em Floresta Ombrófila Mista Montana. Floresta \& Ambiente. n. 24. 1-12 p. 2017. DOI: http://dx.doi.org/10.1590/21798087.097014.

MELO, F. V.; BROWN, G. G.; CONSTANTINO, R.; LOUZADA, J. N. C.; LUIZÃO, F. J.; MORAIS, J. W.; ZANETTI, R. A importância da meso e macrofauna do solo na fertilidade e como biondicadores. Boletim Informativo da SBCS. jan - abril. 38-42 p. 2009.

MEDEIROS, E. J. T.; CAVALCANTE, F. G.; SILVA, M. A.; SILVEIRA, S. C.; MARTINS, C. M. Diversidade cultura de Cepas de actinobactérias do semiárido. Enciclopedia Biosfera. v.15 n.27. 2018. DOI: 10.18677/EnciBio_2018A87

MENEZES C.E.G.; BROWN G.G.; CONSTANTINO R., LOUZADA, J.N.C.; LUIZÃO F.J.; MORAIS J.W.DE.; ZANETTI R. Macrofauna edáfica em estágios sucessionais de floresta estacional semidecidual e pastagem mista em Pinheiral (RJ). Revista Brasileira de Ciência do Solo .33: 1647- 1656. 2009.

DOI: http://dx.doi.org/10.1590/S0100-06832009000600013 
MERLIM, A. O. Macrofauna edáfica em ecossistemas preservados e degradados de Araucária no Parque Estadual de Campos do Jordão, SP. Dissertação de Mestrado - Escola Superior de Agricultura Luiz de Queiroz da Universidade de São Paulo. 2005.

MOÇO, M. K. S.; GAMA-RODRIGUES, E. F.; GAMA-RODRIGUES, A. C.; CORREIA, M. E. Caracterização da fauna edáfica em diferentes coberturas vegetais na região norte fluminense. Revista Brasileira de Ciência do Solo. V. 29, p. 555564. 2005. DOI: http://dx.doi.org/10.1590/S0100-06832005000400008.

MORSELLI, T. B. G. A. Biologia do solo. Ed. Universitária, UFPel, Pelotas, 2009.

MUELLER, K. E.; HOBBIE, S. E.; CHOROVER, J.; REICH, P. B.; EISENHAUER, N.; et al.,; J. Effects of litter traits, soil biota, and soil chemistry on soil carbon stocks at a common garden with 14 tree species. Biogeochemistry Letters. 2015. DOI: 10.1007/s10533-015-0083-6

PINHEIRO, F. J.; MARTINS, C. M.; FIALHO, J. S.; CORREIA, E. F.; CASCON, P Caracterização da macrofauna edáfica na interface solo-serapilheira em uma área de Caatinga do Nordeste Brasileiro. Enciclopédia Biosfera. v.10 n.19. 2014. Disponível

em: http://www.conhecer.org.br/enciclop/2014b/CIENCIAS\%20BIOLOGICAS/caracteriza cao\%20da\%20macrofauna.pdf

POMPEO, P. N.; DOS SANTOS, M. A. B.; BIASI, J. P.; SIQUEIRA, S. F.; ROSA, M. G.; BARETTA, C. R. D. M. Fauna e sua relação com atributos edáficos em Lages, Santa Catarina - Brasil. Scientia Agraria. V. 17, n. 1. 42-51 p. 2016. Disponível em:https://dialnet.unirioja.es/descarga/articulo/6115675.pdf

RODERJAN, C.V.; GALVÃO, F.; KUNIYOSHI, Y.S.; HATSCHBACH, G.G. As unidades fitogeográficas do Estado do Paraná. Ciência e Ambiente. 24: 1, 75-42, 2002. Disponível em:

http://www.itcg.pr.gov.br/arquivos/File/Produtos_DGEO/Mapas_ITCG/PDF/Mapa_Fit ogeografico A3.pdf

RODRIGUES, W.C. Dives - Diversidade de Espécies - Guia do Usuário. Seropédica: Entomologistas do Brasil. 9p. Disponível em: http://www.ebras.bio.br/dives/. 2007.

ROSA, M. G.; KLAUBERG FILHO, O.; BARTZ, M. L. C.; MAFRA, A. L.; SOUSA, J. P. F.; BARETTA, D. Macrofauna edáfica e atributos físicos e químicos em sistemas de uso do solo no planalto catarinense. Revista Brasileira de Ciência do Solo. v. 39. 1544-1553 p. 2015. DOI: 10.1590/01000683rbcs20150033

SCORIZA, R. N.; CORREIA, M. E. F. Soil Fauna as Indicator in Slope Forest Fragments. Floresta e Ambiente. V. 23, n. 4. 2016.

TRIANA, P. S.; ROUSSEAU, G. X.; PIEDADE, A. R.; CELENTANO, 
D.; ZELARAYÁN, M. L. C. BRAUN, H. La macrofauna del suelo como indicadora de degradación de bosques ribereños en la amazonia oriental brasileira Pinzón, Stefania Triana. Revista de la Facultad de Agronomía. v. 114 n.1: 49-60 p. 2014. Disponível em: http://revista.agro.unlp.edu.ar/index.php/revagro/article/view/73

VELOSO, H.P.; RANGEL FILHO, A.L.R.; LIMA, L.C.A. Classificação da vegetação brasileira. IBGE. 1991. 